\title{
BMJ Open Association between acculturation and body weight status among migrant children in Guangzhou, China: a cross- sectional study
}

Xiaoling Huang, ${ }^{1,2}$ Wen Chen, ${ }^{1,2}$ Yanwei Lin, ${ }^{1,2}$ Qi Zhang, ${ }^{2,3}$ Li Ling ${ }^{1,2}$

To cite: Huang X, Chen W, Lin Y, et al. Association between acculturation and body weight status among migrant children in Guangzhou, China: a crosssectional study. BMJ Open 2018;8:e018768. doi:10.1136/ bmjopen-2017-018768

- Prepublication history and additional material for this paper are available online. To view these files, please visit the journal online (http://dx.doi. org/10.1136/bmjopen-2017018768).

$\mathrm{XH}$ and WC contributed equally.

Received 19 July 2017

Revised 25 May 2018

Accepted 29 May 2018
Check for updates

${ }^{1}$ Faculty of Medical Statistics and Epidemiology, School of Public Health, Sun Yat-sen University, Guangzhou, China ${ }^{2}$ Sun Yat-sen Center for Migrant Health Policy, Sun Yat-sen University, Guangzhou, China ${ }^{3}$ School of Community and Environmental Health, Old Dominion University, Norfolk, Virginia, USA

Correspondence to

Dr Li Ling;

lingli@mail.sysu.edu.cn

\section{ABSTRACT}

Objective To assess the association between acculturation and body weight status among internal migrant children in China.

Design, setting and participants A cross-sectional study was conducted among 1154 pairs of migrant children aged 11-17 years and their primary caregivers in Guangzhou, China, from April to May 2016.

Measures Migrant children's body weight status was measured by body mass index. Acculturation was measured by a questionnaire, developed and validated by the research team. The questionnaire had three dimensions with five factors, namely language, social interaction, custom, dressing and diet. Social anxiety was measured by Social Anxiety Scale for Children. Food intake was measured by the food frequency table that was developed from a previous study. Logistic regression was performed to examine the association between acculturation and overweight/obesity while controlling for migrant children's and their caregivers' demographic characteristics, children's social anxiety and food intake. Results Seventy-six out of 1154 (6.6\%) migrant children were overweight, and $36(3.1 \%)$ were obese. The overall prevalence of overweight/obesity was $12.5 \%$ in boys, and $6.1 \%$ in girls $(p<0.001)$, respectively. The mean scores of acculturation were $41.8(S D=14.6)$. Migrant children had the highest level of acculturation in the aspect of dressing (mean=61.7, SD=16.6), followed by language (mean=47.9, SD=22.3), diet (mean=45.0, $\mathrm{SD}=18.5$ ), social interaction (mean $=42.4, \mathrm{SD}=21.3$ ) and custom (mean=27.6, SD=19.2). After controlling for confounding factors, the levels of acculturation was negatively associated with overweight/obesity (adjusted OR (aOR)=0.98, 95\% $\mathrm{Cl} 0.97$ to $1.00, \mathrm{p}=0.030$ ). Furthermore, migrant children who had urban-to-urban migrant caregivers were more likely to be overweight/ obese $(\mathrm{aOR}=2.07,95 \% \mathrm{Cl} 1.16$ to $3.69, \mathrm{p}=0.014)$ than their counterparts living with rural-to-urban migrant caregivers.

Conclusions The low levels of acculturation was associated with overweight/obesity among migrant children in Guangzhou, China. Promoting healthy acculturation and social campaign on healthy body weight may help prevent childhood overweight/obesity. Young migrant children, boys and children living with urban-tourban migrant caregivers should be the target subgroups.
Strengths and limitations of this study

- This study controlled mental health, dietary and primary caregivers' migrant background to assess the relationship between acculturation and overweight/ obesity among internal migrant children in a metropolitan area in China.

- One of the first efforts to link acculturation and childhood obesity among migrants in China.

- Self-reported data were used to calculate the body weight status, which may have recall bias and reporting bias.

Association, instead of causality, was examined.

\section{INTRODUCTION}

With the rapid urbanisation and economic reform in China, internal migration has been accelerated since the late 1950s. ${ }^{12}$ Under the permanent household registration (hukou) system in China, resident status is categorised as rural and urban residency. Due to the unbalanced economic development across regions in China, residents move across the country for a better life $\mathrm{e}^{3}$ and form the 247 million (18\%) internal migrant population (2015), and the majority of them are rural-to-urban migrants. ${ }^{4}$ Under the hukou system, the migration process creates social, economic and cultural challenges and barriers to internal migrant population. ${ }^{2}$ For instance, internal migrants have limited access to the local education, public health service and social welfare, which are provided for local residents by governments. ${ }^{2}$

Internal migrant children are generated by tailing with their caregivers who migrated from developing areas to relatively developed areas within China. ${ }^{5}$ They are a critical subgroup of the migrants and experience significant socioeconomic and cultural changes and challenges. ${ }^{5}$ Chinese census data showed that internal migrant children aged $0-17$ years old had increased from 2.45 million in 2000 to 
35.81 million in 2010 , accounted for $12.84 \%$ of children in China. ${ }^{67}$ Under the hukou system, internal migrant children were defined as those younger than 18 years old and living in a place, rather than registered permanent residence for at least 6 months. ${ }^{2} 6$

Worldwide, childhood obesity is an emerging public health concern, because it has been proven as a significantly early life risk factor for adult obesity and chronic diseases. ${ }^{89}$ Based on a systematic analysis of the data from 188 countries and 21 regions, the prevalence of overweight and obesity in developing countries has increased from $8.1 \%$ to $12.9 \%$ for boys and from $8.4 \%$ to $13.4 \%$ for girls from 1980 to 2013 , while the prevalence in developed countries has grown up from $16.9 \%$ to $23.8 \%$ and from $16.2 \%$ to $22.6 \%$ for boys and girls, respectively. ${ }^{10}$ There also have been sustained increases in the rate of overweight/obesity among children aged 7-18 years old in China from 2000 to 2014, especially for boys, rising from $15.68 \%$ to $28.19 \%$ and from $5.88 \%$ to $20.26 \%$ in urban and rural areas, respectively. ${ }^{11}$ However, the body weight status among children varies across regions in China. In general, southern China has a lower prevalence of overweight/obesity than other regions. ${ }^{12}$ Guangzhou, as a metropolis in southern China, had the lower body mass index (BMI) (19.3) among children than other regions like Shanghai (21.0) and Beijing (21.5). ${ }^{11} 13$

The spatial disparity in childhood overweight and obesity has also been observed among internal migrant children. A study in Guangzhou showed the prevalence of overweight/obesity among migrant children aged $5-12$ years was $14.3 \%$ in $2014,{ }^{14}$ which was lower than figures found among migrant children in Shanghai $(21.1 \%) .{ }^{15}$ This healthy body weight may be explained by local geographical environment and life habits that have formed throughout its long history, including diet, language and custom. ${ }^{131617}$ For instance, the traditional Cantonese diet is relatively healthy compared with the diet in most regions of China, ${ }^{18}$ and the diet in Guangzhou is characterised by manifold styles from throughout China and other countries. Besides, language may play an important role to lead to body weight changes among migrant children via affecting life habits and social interaction. ${ }^{19}$

The trend of migrant children's body weight status in China was scanty due to limited available data. However, in high-income countries, immigrant children were susceptible to childhood obesity with an increasing risk over recent years. ${ }^{20}{ }^{21}$ The increasing burden of obesity among immigrant children has been considered to be results of changes in environmental and socioeconomic factors, as well as exposure to obesity-related risk factors during and after resettlement in new obesogenic environments and adaptation to unhealthy behaviours, such as intake of high-calorie food, insufficient exercise and sedentariness. ${ }^{1422}$ Among these reasons, the change of environmental factors plays a vital role in body weight, mediated by energy imbalance and lifestyle changes. ${ }^{23} 24$
Acculturation, as a multifaceted concept, reflects the overall adaptation to a new society when contacting with individuals and groups from another culture, including the complex and dynamic nature of cultural identity, social connection and preferences in the residence culture (eg, language, history, foods and holiday). ${ }^{25} 26$ Particularly, during the migration, the change of environment and adaptation process may influence their diet, physical activity and other life behaviours, which may further be bidirectionally associated with health status. On the one hand, it may contribute to overweight and obesity among migrant children when they formed bonds with natives and adapt to the obesogenic dietary, behaviours and environment in a receiving society. ${ }^{22}$ On the other hand, migration into the healthy environment may reduce the risk of being overweight or obese. As a study showed that the acculturated parents of migrant children were aware of the health issues associated with obesity as received local attitudes and practices of a healthy lifestyle in main society. ${ }^{27}$

In the current study, inconsistent associations between acculturation and childhood obesity among children of immigrants have been reported. For instance, previous literature reported that high levels of acculturation was related to high BMI among adolescents, particularly among immigrant children from developing countries to developed countries. ${ }^{22} 28$ Jennifer and Baker found that immigrant children with low degrees of acculturation were likely to gain weight. ${ }^{29}{ }^{30}$ Moreover, there was also research demonstrating no significant relationship between acculturation and body weight among immigrant children in the USA. ${ }^{31}$ No consistent acculturation measure was used in related studies. For example, Liu and colleagues ${ }^{22}$ used generation status and language preference as measures. Another study measured acculturation by Child Short Acculturation Scale for Hispanics, including language, media and ethnic social relations. ${ }^{30}$ Moreover, these inconsistent results were also explained by the complication of forming cultural values among different study populations. For example, children with various age could have notable differences in the self-evaluation of body image across culture, as well as the views of acculturation might vary person to person who had different cultural background. ${ }^{32}$

Another limitation in previous studies was assessing the association between acculturation and obesity among immigrant children by only controlling for basic demographic characteristics and food intake. ${ }^{33} 34$ However, other factors, such as the number of children in a family, ${ }^{35}$ mental health ${ }^{36-38}$ and the environmental, socioeconomic, cultural and behavioural difference between sending and receiving communities ${ }^{39}$ were also related to acculturation and obesity and should be considered as confounding factors. For instance, previous studies showed that social anxiety had complex relationships with acculturation ${ }^{38}$ and it was associated with high BMI. ${ }^{40}$ The number of children in family might influence the concerns and supervision from 
parents, which might further effect on their adaptation in a new society. ${ }^{35}$

Therefore, the aim of the study was to investigate the association between acculturation and body weight status among migrant children in China by controlling for aforementioned confounding factors.

\section{METHODS}

\section{Study setting}

This cross-sectional study was conducted from April to May 2016 at six private junior high schools funded by social organisations in Tianhe and Baiyun Districts, Guangzhou, China. Guangzhou is the capital city of Guangdong Province that is the primary destination of internal migrants in China and has hosted more than 0.6 million migrant children by $2016 .{ }^{611}$ Under the hukou system in China, migrant children without hukou are widely excluded from accessing public schools in receiving cities. The majority of them have to attend private schools instead. In Guangzhou, private schools are mainly gathered in Tianhe and Baiyun districts. Therefore, using purposive sampling method, we chose three private junior high schools with large numbers of migrant students in each district, respectively.

\section{Study participants}

The study participants were children who were studying in grades 7-8 at one of the six private junior high schools and met the following criteria: (1) do not have hukou in Guangzhou and have lived in Guangzhou for at least 6 months; (2) aged under 18 years old; (3) at least one of their parents does not have hukou in Guangzhou; and (4) the informed consent was signed by child's primary caregivers.

\section{Participants involvement and data collection}

In this study, a self-administered questionnaire was developed by the research team after a literature review and a preliminary survey, and then given to migrant children and their primary caregivers. Migrant children completed the survey under the guidance of investigators, while they were also trained by the investigators to take the caregivers' questionnaires home and help their primary caregivers finish them. After collecting the questionnaires, investigators would check carefully and contact the primary caregivers timely when they found important missing data. Investigators received standardised training by the research team, and quality control was implemented during data collection. If participants had any questions about their result, they would receive feedback.

\section{Ethics statement}

Written informed consents were obtained from all children's caregivers.

\section{MEASUREMENTS}

\section{Children's body weight status}

Body weight status was measured by the BMI based on self-reported weight and height, while the BMI was calculated by the weight divided by the square of height $(\mathrm{kg} /$ $\mathrm{m}^{2}$ ). Migrant children's overweight and obesity were defined based on the norm established by the working group on obesity in China (WGOC) ${ }^{42}$ This WGOC norm uses the 85th percentile and the 95th percentile of BMI within each age-gender subgroup of Chinese children as cut-off points to define overweight and obesity, respectively (online supplementary appendix table A-1). Overweight is defined as having BMI greater than or equal to 85th percentile and lower than 95th percentile of BMI within each gender-age subgroup. Obesity is defined as having BMI greater than or equal to 95th percentile of BMI within each gender-age subgroup. This definition is analogous to Centers for Disease Control and Prevention growth charts in the USA, which was revised from WHO/ NCHS (the US National Center for Health Statistics) standard. ${ }^{22} 43$

\section{Children's acculturation}

Acculturation in this study was measured by a brief, age-appropriate and multidimensional questionnaire developed by the research team. ${ }^{3244-47}$ The acculturation scale for migrant children included 12 items, which were primitively summarised into three dimensions. That is, language (four items), social interaction (two items) and preferences in life (six items). Preferences in life further included three factors, namely custom (two items), dressing (two items) and diet (two items). The response to each item was assigned a score according to the level of acculturation from maintenance of original culture to maintenance of the host culture. Second, the factor analysis generated five factors from original items, namely language, social interaction, custom, dressing and diet (online supplementary appendix table A-2). Meanwhile, five factor scores were weighed by the percent of variance and added up to estimate the dimension scores and the acculturation score. Finally, the scores were further transformed into a $0-100$ scale by the min-max normalisation and percentage method, whereby 0 points represented the lowest level of acculturation and 100 points were optimum. In this study, the reliability test of the overall scale showed the Cronbach's alpha of 0.70. Table 1 showed the acculturation scale for migrant children.

\section{Confounding factors}

\section{Demographic characteristics}

In this study, the demographic characteristics of migrant children and their caregivers included: (1) children's gender, age, years of residence in Guangzhou; (2) caregivers' education and type of migration (rural-to-urban migration and urban-to-urban migration), household monthly income per person and number of children in the family. Children's age were collected as concrete years old and further divided into two groups (11-13 and 
Table 1 The acculturation scale for migrant children in Guangzhou, China, in $2016^{*}$

\begin{tabular}{llll}
\hline Dimension & Factor & Item & Valuation \\
\hline $\begin{array}{l}\text { Language } \\
\text { (Cronbach's alpha=0.81) }\end{array}$ & Language & (1) Can you understand Cantonese? & $1=$ don't understand completely; \\
& & $2=$ understand partly; \\
& & (2) How about do you speak & $1=$ can't; $2=$ not well; $3=$ better; \\
& Cantonese? & $4=$ fluently. \\
& (3) Did you watch Cantonese TV & $1=$ never; 2=once in a while; \\
& show? & $3=$ sometimes; $4=$ often. \\
& 4) Did you listen to the Cantonese & $1=$ never; $2=$ once in a while; \\
& songs? & $3=$ sometimes; $4=$ often.
\end{tabular}

Social interaction

(Cronbach's alpha=0.55)

Preferences in life
(Cronbach's alpha $=0.52$ )
Social interaction

(1) Where did your good friends come from?

(2) If it is up to you, where do you hope your classmates come from?

(1) If it is up to you, would you like to go to hometown for the Spring Festival?

(2) If it is up to you, do you hope how to spend the Spring Festival?

(2) What kind of dishes do you like?

\begin{tabular}{|c|c|c|}
\hline \multirow[t]{2}{*}{ Custom } & $\begin{array}{l}\text { (1) If it is up to you, would you like } \\
\text { to go to hometown for the Spring } \\
\text { Festival? }\end{array}$ & $\begin{array}{l}1=y e s, \text { I would; } 2=\text { it doesn't matter; } \\
3=\text { no, I would not. }\end{array}$ \\
\hline & $\begin{array}{l}\text { (2) If it is up to you, do you hope how } \\
\text { to spend the Spring Festival? }\end{array}$ & $\begin{array}{l}1=\text { completely in way of hometown; } \\
2=\text { =most of way like hometown; } 3=\text { half like } \\
\text { hometown and half like Guangzhou; } \\
4=\text { most of way like Guangzhou; } \\
5=\text { completely in way of Guangzhou. }\end{array}$ \\
\hline \multirow[t]{2}{*}{ Dressing } & $\begin{array}{l}\text { (1) Compared with your hometown, } \\
\text { do you think people's dressing in } \\
\text { Guangzhou is good looking? }\end{array}$ & $\begin{array}{l}1=\text { not good looking; } 2=\text { no difference/ } \\
\text { unaware; } 3=\text { good looking. }\end{array}$ \\
\hline & $\begin{array}{l}\text { (2) If it's up to you, what kind of } \\
\text { dressing do you wear? }\end{array}$ & $\begin{array}{l}1=\text { completely in way of hometown; } \\
2=\text { =most of way like hometown; } 3=\text { half like } \\
\text { hometown and half like Guangzhou; } \\
4=\text { most of way like Guangzhou; } \\
5=\text { completely in way of Guangzhou. }\end{array}$ \\
\hline \multirow[t]{2}{*}{ Diet } & $\begin{array}{l}\text { (1) What kind of food do you usually } \\
\text { eat? }\end{array}$ & $\begin{array}{l}1=\text { all dishes like hometown; } 2=\text { most of } \\
\text { dishes like hometown; } 3=\text { half like } \\
\text { hometown and half like Guangzhou; } \\
4=\text { most of dishes like Guangzhou; } \\
5=\text { =all dishes like Guangzhou. }\end{array}$ \\
\hline & (2) What kind of dishes do you like? & $\begin{array}{l}\text { 1=hometown dishes; } 2=\text { =local dishes; } \\
\text { 3=foreigner dishes. }\end{array}$ \\
\hline
\end{tabular}

*The Cronbach's alpha of reliability analysis on the acculturation scale for children was 0.70 . $2=$ half were non-local friends and half were local friends; $3=$ most of them were local friends.

\section{$1=y e s, I$ would; 2=it doesn't matter;}

$1=$ completelyin way of hometown; $2=$ most of way like hometown; $3=$ half like angzhou; ( 1=notgood looking: 2 =no difference/ $1=$ completely in way of hometown; 2=most of way like hometown; 3=half like angzhou 4=most of way like Guangzhou; $5=$ completely in way of Guangzhou.

$1=$ all dishes like hometown; $2=$ most of dishes like hometown; 3 =half like hometown and half like Guangzhou; $4=$ most of dishes like Guangzhou;

1=hometown dishes; $2=$ =local dishes; igner dishes.
14-17 years old) based on the mean value (mean $=13.8$, $\mathrm{SD}=0.9$ ). The 14 years old is also used as the cut-off point for children and adolescents in China. ${ }^{48}$ Household monthly income per person was defined as a continuous variable and further divided into four categories by the quartiles ( $\leq 3500 \mathrm{RMB}$, 3501-5000 RMB, 5001-8000 RMB and $\geq 8001 \mathrm{RMB}$ ).

\section{Children's social anxiety}

The social anxiety in our survey was measured by Social Anxiety Scale for Children (SASC). ${ }^{49}$ This scale included 10 items yielding two dimensions, including fear of negative evaluation and social avoidance and distress. The response to each item was recorded using a three-point scale from 0 to 2 , with potential scores ranging from 0 to 20. Higher scores represented better mental status in the corresponding psychosocial issues. SASC showed the good psychometric properties with Cronbach's alpha of 0.79 among children in China. ${ }^{50}$ In our study, SASC demonstrated the Cronbach's alpha of 0.82 .

\section{Food intake}

To measure the status of food intake, we used the food frequency table that was developed from the study of dietary factors and endometrial cancer in China. ${ }^{51}$ The respondents' food intake was measured by counting their consumption frequency on 30 foods, which were categorised into four major food groups based on the dietary nutritional perspective: (1) carbohydrate-based food (eg, rice and noodles); (2) animal food (eg, meat, fish and egg); (3) milk, beans and products; and (4) vegetables and fruits. For each food item, participants were asked about how often they ate the food during the past 1 month $(0=$ never or less once a month, $1=$ once or twice 
a month, 2=once a week, 3=2-5 times a week, $4=$ once a day, $5=$ more than twice a day). Then, each item score was added to get the score of each food group, while high scores represented high levels of consumption on corresponding food category.

\section{Statistical analysis}

The statistical analysis was performed using IBM SPSS V.20.0. The categorical and numerical variables were described by frequency, proportion, mean and SD, respectively. $\chi^{2}$ test, rank sum test or independent samples t-test were used for comparisons between overweight or obese migrant children and normal weight migrant children. Paired samples t-test with Bonferroni correction was used to pairwise compare among scores of three dimensions/ five factors of acculturation. Bivariate and multivariate logistic regression analysis were performed to examine the association between acculturation and overweight/ obesity among migrant children while controlling for demographic characteristics of migrant children and their caregivers, children's social anxiety and four categories of food intake. The interaction between acculturation and the demographic characteristics (including migrant children's gender and caregiver's type of migration) was also taken into consideration in the model. Crude ORs, adjusted ORs (aOR), 95\% CIs and $\mathrm{p}$ value were calculated to present the association between acculturation and overweight/obesity.

\section{RESULTS \\ Characteristics of migrant children and their primary caregivers}

There were 1199 pairs of eligible migrant students and their primary caregivers participating in the survey, but $45(3.8 \%)$ migrant students were excluded due to not reporting height and weight. Finally, we included 1154 (96.2\%) pairs of participants and their caregivers in this study.

Table 2 showed 112 (9.7\%) migrant children were overweight/obesity (BMI: mean=25.0, $\mathrm{SD}=3.0$ ), including $76(6.6 \%)$ were overweight and $36(3.1 \%)$ were obese. The average age of migrant children was $13.7 \quad(\mathrm{SD}=0.9)$ years old. There were statistically differences in the prevalence of childhood overweight/obesity between different subgroups of gender, age and caregivers' type of migration, respectively $(\mathrm{p}<0.05)$. There were $12.5 \%(81 / 647)$ of boys and $6.1 \%(31 / 507)$ of girls being overweight and obese. Children who were 13 years or younger had a higher prevalence of overweight/obesity $(13.9 \%, 66 / 476)$ than those aged 14 years and over $(6.8 \%, 46 / 678)$. Furthermore, the prevalence of overweight/obesity was higher among migrant children whose primary caregivers with urban-to-urban migration background $(14.6 \%, 83 / 955)$ than those living with caregivers with rural-to-urban migration $(8.7 \%, 27 / 185)$.

\section{Acculturation status of migrant children}

Table 3 presented the acculturation score and scores of each dimension and each factor. Among all migrant children, the average acculturation score was $41.8(\mathrm{SD}=14.6)$ out of 100 . There were no statistically differences in acculturation and scores of dimensions and factors of acculturation between overweight/obese migrant children and normal weight migrant children $(\mathrm{p}=0.067)$. Among all migrant children, the scores of language (mean difference $=5.5$, $\mathrm{SD}=30.6$ ) and preferences in life (mean difference $=4.9$, $\mathrm{SD}=25.4$ ) were significantly higher than the score of social interaction $(p<0.001)$. In terms of factor scores, migrant children had the highest level of acculturation in the aspect of dressing (mean=61.7, $\mathrm{SD}=16.6$ ), followed by language $($ mean $=47.9, \mathrm{SD}=22.3)$, diet $($ mean $=45.0$, $\mathrm{SD}=18.5)$, social interaction (mean $=42.4, \mathrm{SD}=21.3)$ and custom (mean=27.6, $\mathrm{SD}=19.2)(\mathrm{p}<0.001)$. However, differences in scores of dimensions and factors of acculturation between overweight/obese migrant children and normal weight migrant children were not significant.

Association between children's acculturation and overweight/ obesity

Table 4 represented the results of logistic regression models on the association between acculturation and overweight/obesity among migrant children. After controlling for demographic characteristics of migrant children and their primary caregivers, children's social anxiety and food intake, the levels of acculturation were negatively associated with their overweight/obesity $(\mathrm{aOR}=0.98,95 \%$ CI 0.97 to $1.00, \mathrm{p}=0.030)$. Furthermore, compared with rural-to-urban migrated caregivers, caregivers with urban-to-urban migration background were more likely to increase the likelihood of their children's overweight/obesity (aOR=2.07, 95\% CI 1.16 to 3.69, $\mathrm{p}=0.014$ ).

\section{DISCUSSION}

Childhood overweight and obesity are a major global public health crisis for the health and well-being of both children and adults. ${ }^{9}$ Migrant children face more challenges in health issues than their local counterparts. ${ }^{5}$ The prevalence of overweight $(6.6 \%)$ and obesity $(3.1 \%)$ found in this study were lower than that among immigrant children in developed countries (over 10.0\% and over $15.0 \%) .{ }^{20252}$ Internal migrant children had a lower prevalence of overweight/obesity than immigrant children may be mainly due to different diet cultures between China and developed countries. ${ }^{53}$ The prevalence of obesity in this study $(3.1 \%)$ was close to that among students aged $12-18$ years $(3.4 \%)$ in Guangzhou (2013), ${ }^{13}$ in which children had lower BMI than those in other cities. However, the prevalence of obesity among migrants in this age group in other regions of China was scanty. Meanwhile, it is notable that China is a vast country with diversity, and children in various regions have different prevalence of obesity (girls: $0.44 \%-10.00 \%$ vs boys: $0.56 \%-21.67 \%) .{ }^{5455}$ The spatial, diet and cultural disparity within China may be the significant reasons of differences in obesity rates and need to be further explored. ${ }^{13} 17$ 
Table 2 Characteristics of 1154 migrant children and their primary caregivers in Guangzhou, China, in 2016

\section{Overweight/obesity}

Characteristic

No† Yes $¥$

Total

Children's characteristic

Gender ( $n=1154), n$ (\%)

$\begin{array}{lccc}\text { Male } & 566(87.5) & 81(12.5) & 647(56.1)^{\star \star *} \\ \text { Female } & 476(93.9) & 31(6.1) & 507(43.9) \\ \text { Age }(\mathrm{n}=1154) \text { (years), mean } \pm \text { SD } & 13.8 \pm 0.9 & 13.4 \pm 0.9 & 13.7 \pm 0.9^{\star \star \star} \\ 11-13, \mathrm{n}(\%) & 410(86.1) & 66(13.9) & 476(41.2) \\ 14-17, \mathrm{n}(\%) & 632(93.2) & 46(6.8) & 678(58.8) \\ \text { Years of residence in Guangzhou ( } \mathrm{n}=1103), \mathrm{n}(\%) & & & \\ <6 & 238(91.5) & 22(8.5) & 260(23.6) \\ 6-10 & 289(91.2) & 28(8.8) & 317(28.7) \\ 11-16 & 470(89.4) & 56(10.6) & 526(47.7) \\ \text { Social Anxiety Scale for Children }(\mathrm{n}=1108), \text { mean } \pm \text { SD } & 14.2 \pm 3.9 & 14.5 \pm 3.9 & 14.2 \pm 3.9 \\ \text { Fear of negative evaluation } & 8.5 \pm 2.6 & 8.6 \pm 2.5 & 8.5 \pm 2.6 \\ \text { Social avoidance and distress } & 5.7 \pm 1.9 & 5.9 \pm 1.8 & 5.7 \pm 1.9 \\ \text { Food intake ( } \mathrm{n}=1131), \text { mean } \pm \text { SD } & 67.1 \pm 21.9 & 66.4 \pm 22.5 & 67.0 \pm 21.0 \\ \text { Carbohydrate-based food } & 14.9 \pm 3.7 & 14.5 \pm 4.1 & 14.9 \pm 3.7 \\ \text { Animal food } & 29.7 \pm 13.6 & 29.4 \pm 14.4 & 29.7 \pm 13.7 \\ \text { Milk, beans and products } & 14.0 \pm 6.9 & 14.3 \pm 6.8 & 14.1 \pm 6.9 \\ \text { Vegetables and fruits } & 8.5 \pm 1.6 & 8.3 \pm 2.0 & 8.4 \pm 1.6\end{array}$

Primary caregivers' characteristic

Caregivers' education ( $n=1100), n(\%)$

Primary school and below (<grade 6)

Middle school (grades 7-9)

$151(92.1)$

$573(90.5)$

$13(7.9)$

$164(14.9)$

High/vocational school (grades 10-12)

$218(90.0)$

$60(9.5)$

$633(57.5)$

Junior college and above (grade 12-)

$53(91.4)$

$27(11.0)$

$245(22.3)$

Caregivers' type of migration ( $n=1140), n(\%)$

\begin{tabular}{|c|c|c|c|}
\hline Rural-to-urban migration & $872(91.3)$ & $83(8.7)$ & $955(83.8)^{*}$ \\
\hline Urban-to-urban migration & $158(85.4)$ & $27(14.6)$ & $185(16.2)$ \\
\hline \multicolumn{4}{|c|}{ Household monthly income per person $(n=1140), n(\%)$} \\
\hline$\leq 3500 \mathrm{RMB}$ & $259(90.9)$ & $26(9.1)$ & $285(25.0)$ \\
\hline $3501-5000 \mathrm{RMB}$ & 306 (87.9) & $42(12.1)$ & $348(30.5)$ \\
\hline 5001-8000 RMB & $225(90.4)$ & $24(9.6)$ & $249(21.8)$ \\
\hline$\geq 8001 \mathrm{RMB}$ & $240(93.0)$ & $18(7.0)$ & $258(22.7)$ \\
\hline \multicolumn{4}{|c|}{ Number of children in family ( $n=1133), n(\%)$} \\
\hline 1 & $121(87.7)$ & $17(12.3)$ & $138(12.2)$ \\
\hline 2 & $459(89.6)$ & $53(10.4)$ & $512(45.2)$ \\
\hline$\geq 3$ & $445(92.1)$ & $38(7.9)$ & $483(42.6)$ \\
\hline
\end{tabular}

Comparison the prevalence of overweight/obesity between different subgroups of each categorical variable. ${ }^{*} P<0.05 ;{ }^{* \star} p<0.01 ;{ }^{* \star *} p<0.001$. †BMI: mean=17.8, $S D=2.1$.

łBMI: mean=25.0, SD=3.0.

$\mathrm{BMI}$, body mass index.

In this study, the acculturation status among internal migrant children was not high, but the highest score of acculturation was observed in the aspect of dressing, followed by language, diet, social interaction and custom.
As the development of modern media technique, the mass and abundant media has been a public educator and affected our attitudes in fashion, dressing and health exercise $^{56}$ especially among rural-to-urban children. 
Table 3 Acculturation score and scores of dimensions and factors of acculturation among 1154 migrant children in Guangzhou, China, in 2016

\begin{tabular}{|c|c|c|c|c|c|}
\hline \multirow[b]{2}{*}{ Acculturation } & \multicolumn{3}{|c|}{ Overweight/obesity, mean \pm SD } & \multirow[b]{2}{*}{$P_{1}$} & \multirow[b]{2}{*}{$\boldsymbol{P}_{2}$} \\
\hline & No & Yes & Total & & \\
\hline Acculturation & $42.0 \pm 14.7$ & $39.4 \pm 13.5$ & $41.8 \pm 14.6$ & 0.067 & - \\
\hline Language & $48.3 \pm 22.2$ & $44.7 \pm 23.4$ & $47.9 \pm 22.3$ & 0.095 & - \\
\hline Social interaction & $42.4 \pm 21.4$ & $42.8 \pm 20.4$ & $42.4 \pm 21.3$ & 0.607 & - \\
\hline Preferences in life & $47.5 \pm 14.0$ & $45.6 \pm 12.4$ & $47.3 \pm 13.9$ & 0.294 & - \\
\hline Custom & $27.6 \pm 19.2$ & $27.1 \pm 18.9$ & $27.6 \pm 19.2$ & 0.852 & - \\
\hline Dressing & $61.9 \pm 16.7$ & $59.9 \pm 15.8$ & $61.7 \pm 16.6$ & 0.194 & - \\
\hline Diet & $45.2 \pm 18.4$ & $43.6 \pm 19.6$ & $45.0 \pm 18.5$ & 0.483 & - \\
\hline \multicolumn{6}{|c|}{ Differences between dimensions $\left(\alpha^{\prime}=0.05 / 3\right)$} \\
\hline Language: social interaction & $5.9 \pm 30.6$ & $1.9 \pm 30.7$ & $5.5 \pm 30.6$ & 0.207 & $<0.001$ \\
\hline Language: preferences in life & $0.8 \pm 26.0$ & $-0.9 \pm 27.5$ & $0.6 \pm 23.2$ & 0.414 & 0.430 \\
\hline Preferences in life: social interaction & $5.1 \pm 25.5$ & $2.8 \pm 24.6$ & $4.9 \pm 25.4$ & 0.390 & $<0.001$ \\
\hline \multicolumn{6}{|l|}{ Differences between factors $\left(\alpha^{\prime}=0.05 / 10\right)$} \\
\hline Language: social interaction & $5.9 \pm 30.6$ & $1.9 \pm 30.7$ & $5.5 \pm 30.6$ & 0.207 & $<0.001$ \\
\hline Language: custom & $20.6 \pm 29.5$ & $17.6 \pm 29.8$ & $20.3 \pm 29.6$ & 0.222 & $<0.001$ \\
\hline Language: dressing & $-13.6 \pm 27.7$ & $-15.2 \pm 28.1$ & $-13.7 \pm 27.7$ & 0.547 & $<0.001$ \\
\hline Language: diet & $3.1 \pm 28.4$ & $1.1 \pm 33.0$ & $2.9 \pm 28.8$ & 0.544 & 0.001 \\
\hline Social interaction: custom & $14.7 \pm 28.7$ & $15.7 \pm 28.8$ & $14.8 \pm 28.7$ & 0.804 & $<0.001$ \\
\hline Social interaction: dressing & $-19.5 \pm 27.1$ & $-17.1 \pm 25.4$ & $-19.3 \pm 26.9$ & 0.330 & $<0.001$ \\
\hline Social interaction: diet & $-2.8 \pm 28.2$ & $-0.8 \pm 29.1$ & $-2.6 \pm 28.3$ & 0.546 & 0.003 \\
\hline Custom: dressing & $-34.2 \pm 25.3$ & $-32.8 \pm 25.6$ & $-34.1 \pm 25.3$ & 0.575 & $<0.001$ \\
\hline Custom: diet & $-17.5 \pm 26.6$ & $-16.5 \pm 27.8$ & $-17.4 \pm 26.7$ & 0.845 & $<0.001$ \\
\hline Dressing: diet & $16.7 \pm 24.8$ & $16.3 \pm 27.4$ & $16.6 \pm 25.1$ & 0.761 & $<0.001$ \\
\hline
\end{tabular}

$P_{1}$ is the significance of the comparison between overweight/obesity migrant children and normal weight migrant children.

$P_{2}$ is the significance of pairwise comparisons among dimensions or factors of acculturation. Bonferroni was used to adjust $\alpha\left(\alpha^{\prime}\right)$ to pairwise compare.

Language affects the communication between migrants and local residents, which may have a further impact on social interaction and enable migrant children to engage in activities with local children. ${ }^{19}$ However, under the influence of Chinese traditional culture, original culture has a profound impact on custom, particularly which is derived from some important festivals like spring festival.

Our study indicated that internal migrant children with higher levels of acculturation were less likely to be overweight/obesity than those with lower levels of acculturation. This finding was contrary to previous studies conducted in developed countries like USA, which found a positive correlation between the degree of acculturation and overweight/obesity among immigrant children. ${ }^{22} 28$ First, different diet cultures among receiving societies could be a possible explanation. Guangzhou is famous for its healthy Cantonese diet culture ${ }^{18}$; for example, urban children in Guangzhou had a lower prevalence of overweight/obesity than their counterparts in other areas in China. ${ }^{16}$ Hence, in this study, migrant children having high levels of acculturation were more likely to integrate into Guangzhou's eating environment and keep fitness. On the contrary, in developed countries, acculturated immigrant children were more likely to adapt to the obesogenic environment. ${ }^{57}$ However, it is notable that other obesity-related behaviours (eg, consumption of fast food and longer hours of media playing) also exist among children in Guangzhou and other multicultural metropolis. ${ }^{56}$ Therefore, health policy and social campaign on healthy body weight should be advocated among migrant children and targeting their local counterparts. Second, it is noteworthy that migrant children in this study had high degrees of acculturation of dressing. Recent studies indicated that the mainstream culture's obsession with thinness and beauty had a significant impact on body image, which was strongly connected to the weight status of adolescents. ${ }^{58}$ Therefore, with the propaganda of ideal body image in the newly emerging media, migrant children, especially girls, could keep a low weight to pursue fashion and pleasing appearance. ${ }^{59}$ Third, acculturation in language and social interaction both promoted communication with local children to attend activities together including physical activities, which would protect migrant children from overweight and obesity. ${ }^{19}$ 
Table 4 Logistic model on the association between acculturation and overweight/obesity among 1154 migrant children in Guangzhou, China, 2016

\begin{tabular}{|c|c|c|c|c|}
\hline \multirow[b]{2}{*}{ Variable } & \multicolumn{2}{|l|}{ Model 1} & \multicolumn{2}{|l|}{ Model 2} \\
\hline & OR $(95 \% \mathrm{Cl})$ & $P$ values & aOR $(95 \% \mathrm{Cl})$ & $P$ values \\
\hline Acculturation & 0.99 (0.98 to 1.00$)$ & 0.078 & 0.98 (0.97 to 1.00$)$ & 0.030 \\
\hline \multicolumn{5}{|l|}{ Gender } \\
\hline Male (reference) & 1 & & 1 & \\
\hline Female & 0.46 (0.30 to 0.70$)$ & $<0.001$ & $0.43(0.25$ to 0.74$)$ & 0.002 \\
\hline \multicolumn{5}{|l|}{ Age (years) } \\
\hline 11-13 (reference) & 1 & & 1 & \\
\hline $14-17$ & 0.45 (0.30 to 0.67$)$ & $<0.001$ & 0.41 (0.25 to 0.66$)$ & $<0.001$ \\
\hline \multicolumn{5}{|l|}{ Caregivers' type of migration } \\
\hline Rural-to-urban migration (reference) & 1 & & 1 & \\
\hline Urban-to-urban migration & 1.80 (1.13 to 2.86$)$ & 0.014 & 2.07 (1.16 to 3.69$)$ & 0.014 \\
\hline Years of residence in Guangzhou & 1.15 (0.89 to 1.48$)$ & 0.287 & 1.25 (0.91 to 1.73$)$ & 0.166 \\
\hline Fear of negative evaluation & 1.01 (0.94 to 1.09$)$ & 0.743 & 0.95 (0.85 to 1.05$)$ & 0.306 \\
\hline Social avoidance and distress & 1.07 (0.96 to 1.20$)$ & 0.216 & $1.12(0.96$ to 1.30$)$ & 0.149 \\
\hline Carbohydrate based food & 0.97 (0.92 to 1.03$)$ & 0.298 & 0.95 (0.88 to 1.02$)$ & 0.145 \\
\hline Animal food & 1.00 (0.98 to 1.01$)$ & 0.798 & 0.99 (0.97 to 1.02$)$ & 0.602 \\
\hline Milk to beans and products & 1.01 (0.98 to 1.03$)$ & 0.749 & $1.02(0.98$ to 1.07$)$ & 0.312 \\
\hline Vegetables and fruits & 0.94 (0.84 to 1.05$)$ & 0.283 & 0.91 (0.78 to 1.06$)$ & 0.225 \\
\hline Caregivers' education & $1.10(0.84$ to 1.44$)$ & 0.473 & 0.99 (0.71 to 1.38$)$ & 0.951 \\
\hline Household monthly income per person & 0.90 (0.75 to 1.08$)$ & 0.259 & 0.89 (0.71 to 1.12$)$ & 0.330 \\
\hline Number of children in family & 0.77 (0.58 to 1.03$)$ & 0.076 & 1.06 (0.74 to 1.52$)$ & 0.755 \\
\hline
\end{tabular}

Model 1 was a bivariate logistic regression model. Model 2 was a multivariate logistic model adjusted for gender, age, years of residence in Guangzhou, caregivers' education, caregivers' type of migration, household monthly income per person, number of children in family, FNE, SAD and four categories of food intake.

The interaction effect between acculturation and migrant children's gender/caregiver's type of migration were not significant. The model with the interaction was not reported in the table 4.

aOR, adjusted OR; FNE, fear of negative evaluation; SAD, social avoidance and distress.

Therefore, our findings suggested that further health-related acculturation services should focus on promoting healthy body weight and on forming healthy views on body image among children. However, it is notable that developing a healthy body image should be highlighted on the healthy body weight and health consequences, rather than focusing on heavy children's weight-related pressures and stigmatisation. ${ }^{58} 60$

Besides, our study showed that younger migrant children aged 11-13 years had a higher prevalence of overweight/obesity than elder children, and boys had a higher prevalence of overweight/obesity than girls, which agrees with other research findings. ${ }^{23} 29$ Younger migrant children may have poor self-control, especially in unhealthy snacking, like energy-dense and nutrient-poor foods, which could contribute uncompensated calories to overweight/obesity. ${ }^{2461}$ Additionally, the difference of gender on body weight may be explained by girls paying more attention to pleasing appearance than boys. ${ }^{59}$ Moreover, migrant parents place much stricter supervision on their daughters than their sons ${ }^{50}$ and maybe faster to notice the risks of obesity for their daughters than their sons. ${ }^{29}$
Hence, young and male migrant children should be the target subgroups in further research and health promotion of childhood overweight and obesity.

In our study, we also found that migrant children whose caregivers with rural-to-urban background had a lower likelihood of overweight/obesity than those living with urban-to-urban migrant caregivers. It was pointed that the prevalence of overweight and obesity in adults in the city was higher than that in rural areas. ${ }^{62}$ Some studies demonstrated that parental weight status, behaviours and acculturation impacted children's body weight, ${ }^{43} 6364$ which were associated with the culture of their caregiver's place of birth. ${ }^{19}$ Furthermore, China is a country of great cultural diversity, and the differences exist between urban and rural areas and between regions, for example, southern, northern, western and eastern China have very different cultures. ${ }^{17}$ Therefore, future research and practice should pay attention to the caregiver's role in migrant childhood overweight and obesity prevention.

However, there were some limitations of the current study. First of all, although a self-designed multidimensional scale measured acculturation with the acceptable 
overall Cronbach's alpha of 0.70 , two dimensions were having Cronbach's alpha of 0.52 and 0.55 . It indicated the reliability of measurement was reluctantly accepted but need to be further improved. Future research may improve the reliability of the developed acculturation scale by modifying some items. Second, one confounding factor, physical exercise, was not included in this study. ${ }^{28}$ Children's physical exercise included physical activities at school and after school. We only measured physical activities at school, but no differences between overweight/ obese and normal weight migrant children were found. Based on a study in Guangzhou, the variation of extracurricular physical activities of middle school students was small, and all of the students (97.25\%) participated in extracurricular physical activities from Monday to Friday. ${ }^{65}$ Comprehensive measurements on exercise should be considered in the future. Moreover, this study focused on body weight status among migrant children in Guangzhou and did not collect data among local children. However, figures on body weight status among local children in Guangzhou are scanty. Further empirical evidence on the difference between local and migrant children is needed. Finally, it was the limitation of a cross-sectional study that results in selection bias, recall bias and reporting bias based on self-reported data and the determination of association rather than causality.

\section{CONCLUSIONS}

We found that the low levels of acculturation was associated with overweight/obesity among migrant children in Guangzhou, China. Also, the findings suggested that young migrant children, boys and children living with caregivers with urban-to-urban migration background should be the target subgroups in further research and health promotion of childhood overweight and obesity. Importantly, further healthy acculturation services and social campaign on healthy body weight may help prevent childhood overweight and obesity.

\section{Acknowledgements The survey was supported by Sun Yat-sen Center for Migrant Health Policy and the six investigated schools. We would like to thank all investigated students and their primary caregivers for kind cooperation and support. We would also like to thank all teachers and investigators for assistance in data collection. We are also grateful to all who gave valuable comments and suggestions on this paper.}

Contributors XH took part in data analysis and wrote the manuscript. WC contributed to the revisions of the manuscript. YL participated in study design and data collection. QZ contributed to design the project. LL contributed to the supervision on project conducting. All authors read and approved the final manuscript.

Funding This work was supported by China Medical Board grant number (12-111). QZ was supported by the 111 Project (Grant Number B16031).

Competing interests None declared.

Patient consent Parental/guardian consent obtained.

Ethics approval This study was approved by the Institutional Review Board (IRB) of the School of Public Health, Sun Yat-sen University in China ((2015) No.42).

Provenance and peer review Not commissioned; externally peer reviewed. Data sharing statement № additional data are available.
Open access This is an open access article distributed in accordance with the Creative Commons Attribution Non Commercial (CC BY-NC 4.0) license, which permits others to distribute, remix, adapt, build upon this work non-commercially, and license their derivative works on different terms, provided the original work is properly cited and the use is non-commercial. See: http://creativecommons.org/ licenses/by-nc/4.0/

(C) Article author(s) (or their employer(s) unless otherwise stated in the text of the article) 2018. All rights reserved. No commercial use is permitted unless otherwise expressly granted.

\section{REFERENCES}

1. Gui Y, Berry JW, Zheng Y. Migrant worker acculturation in China. Int $J$ Intercult Relat 2012;36:598-610.

2. Mou J, Griffiths SM, Fong HF, et al. Defining migration and its health impact in China. Public Health 2015;129:1326-34.

3. Wang Z, Wang R. Coefficient of family relationship for China's temporary migrant and its determinant. Population \& Economics 2011:9-16.

4. National Health and Family Planning commission. Report on China's migrant population development in 2016. Beijing, China: China Population Publishing House, 2016.

5. Wang L, Mesman J. Child development in the face of rural-to-urban migration in China: a meta-analytic review. Perspect Psychol Sci 2015;10:813-31.

6. Chengrong D, Lidan L, Zongping W, et al. The survival and development status of floating children in China: an analysis of the sixth population census data. South China Population 2013;28:44-55

7. National Bureau of Statistics of the People's Republic of China. Data of fifth census. 2000. http://www.stats.gov.cn/tjsj/ndsj/renkoupucha/ 2000pucha/html//0705.htm

8. Kovesdy CP, Furth SL, Zoccali C. Obesity and kidney disease: hidden consequences of the epidemic. J Ren Care 2017;43:3-10.

9. Singh AS, Mulder C, Twisk JWR, et al. Tracking of childhood overweight into adulthood: a systematic review of the literature. Obesity Reviews 2008;9:474-88.

10. $\mathrm{Ng} \mathrm{M}$, Fleming $\mathrm{T}$, Robinson $\mathrm{M}$, et al. Global, regional, and national prevalence of overweight and obesity in children and adults during 1980-2013: a systematic analysis for the global burden of disease study 2013. The Lancet 2014;384:766-81.

11. State General Administration of Sports of the People's Republic of China. Report on the physical fitness and health survey among Chinese students in 2014. Beijing, China: Higher Education Press, 2016.

12. Cy J, Sun JL. Geographic and population difference of BMI in Chinese school-age youth. Chinese Journal of Pediatrics 2004;42:328-32.

13. Mai J. A study on the relationship between obesity and dietary behaviors, physical activities among adolescents: Taking some students in Guangzhou China as an example Sichuan Normal University. 2014.

14. Liu W, Liu W, Lin R, et al. Socioeconomic determinants of childhood obesity among primary school children in Guangzhou, China. BMC Public Health 2016;16:482.

15. Jk L, Yin XJ, Xiong JP, et al. Comparison of the status of overweight/ obesity among the youth of local Shanghai, young rural-tourban migrants and immigrant origin areas. Int $J$ Clin Exp Med 2015;8:2804-14.

16. Zhao G, He W. Investigation on overweight and obesity of urban children and teenager in Guangzhou. China Sport Science and Technoligy 2008;44:103-6.

17. , Song F, Cho MS; Mi Cho. Geography of Food Consumption Patterns between South and North China. Foods 2017;6:34.

18. Xue W. Cantonese cuisine: the perfect unity of nutritious food and cultural custom. Science and Technology of West China 2005:17-22.

19. Renzaho AMN, Oldroyd J, Burns C, et al. Over and undernutrition in the children of Australian immigrants: Assessing the influence of birthplace of primary carer and English language use at home on the nutritional status of 4-5-year-olds. Int $J$ Pediatr Obes 2009;4:73-80.

20. Gualdi-Russo E, Zaccagni L, Manzon VS, et al. Obesity and physical activity in children of immigrants. Eur J Public Health 2014;24(suppl 1):40-6.

21. Lu W, Jacobs W, Mckyer ELJ. Health risk factors for overweight and obesity among Chinese American children: a systematic review of literature. Health Behav \& Pub Health 2014;4:29-36. 
22. Liu J-H, Chu YH, Frongillo EA, et al. Generation and acculturation status are associated with dietary intake and body weight in Mexican American adolescents. J Nutr 2012;142:298-305.

23. Jc Y, Jiao CT. Empirical changes in the prevalence of overweight and obesity among Chinese students from 1985 to 2010 and corresponding preventive strategies. Biomed Environ Sci 2013;26:1-12.

24. Nishtar S, Gluckman P, Armstrong T. Ending childhood obesity: a time for action. The Lancet 2016;387:825-7.

25. Barry DT, Garner DM. Eating concerns in East Asian immigrants: relationships between acculturation, self-construal, ethnic identity, gender, psychological functioning and eating concerns. EatWeight Disord 2000;6:90-8.

26. Unger JB, Ritt-Olson A, Wagner K, et al. A comparison of acculturation measures among hispanic/latino adolescents. $J$ Youth Adolesc 2007;36:555-65.

27. Chen J-L, Weiss S, Heyman MB, et al. Risk factors for obesity and high blood pressure in chinese american children: maternal acculturation and children's food choices. J Immigr Minor Health 2011;13:268-75

28. Liu J, Probst JC, Harun N, et al. Acculturation, physical activity, and obesity among Hispanic adolescents. Ethn Health 2009;14:509-25.

29. Jv H, Baker E. Big boys and little girls: gender, acculturation, and weight among young children of immigrants. J Health Soc Behav 2010;51:200-14.

30. Moreno JP, Vaughan E, Hernandez D, et al. The impact of acculturation level on weight status and weight outcomes in hispanic children. J Racial Ethn Health Disparities 2016;3:582-9.

31. Fialkowski MK, Ettienne R, Shvetsov YB, et al. Ethnicity and acculturation: do they predict weight status in a longitudinal study among asian, hispanic, and non-hispanic white early adolescent females? Adolesc Health Med Ther 2015;6:1-7.

32. Unger JB, Gallaher P, Shakib S, et al. The AHIMSA Acculturation scale: a new measure of acculturation for adolescents in a multicultural society. Journal of Early Adolescence 2001;22:225-51.

33. Wiley JF, Cloutier MM, Wakefield DB, et al. Acculturation determines bmi percentile and noncore food intake in hispanic children. J Nutr 2014;144:305-10.

34. Wojcicki JM, Schwartz N, Jiménez-Cruz A, et al. Acculturation, dietary practices and risk for childhood obesity in an ethnically heterogeneous population of latino school children in the San Francisco bay area. J Immigr Minor Health 2012;14:533-9.

35. Wang Z, Lin X, Fang X. A comparative study of migrant children's urban adaptation in public schools and schools for migrant workers' children. Chinese Journal of Special Education 2010:21-6.

36. Brewer-Smyth K, Cornelius M, Pohlig RT. Childhood adversity and mental health correlates of obesity in a population at risk. $J$ Correct Health Car 2016;22:367-82.

37. Wh L, Diep CS, McKyer ELJ. Risk factors for childhood obesity among Asian Americans: a systematic review of literature and recommendations for health care research. J Health Care Poor $U$ 2015;26:171-90.

38. Huang K-Y, Cheng S, Calzada E, et al. Symptoms of anxiety and associated risk and protective factors in young asian american children. Child Psychiatry \& Human Development 2012;43:761-74.

39. Ebrahim S, Kinra S, Bowen L, et al. The effect of rural-to-urban migration on obesity and diabetes in india: a cross-sectional study. PLoS Med 2010;7:e1000268.

40. Rankin J, Matthews L, Cobley S, et al. Psychological consequences of childhood obesity: psychiatric comorbidity and prevention. Adolesc Health Med Ther 2016;7:125-46.

41. Xu Q. Nearly 4.3 million migrant children study in Guangzhou and eighty percents are in the pearl river delta. http://www.chinanews. com/sh/2016/01-27/7735970.shtm

42. Group of China Obesity Task Force. [Body mass index reference norm for screening overweight and obesity in Chinese children and adolescents]. Zhonghua Liu Xing Bing Xue Za Zhi 2004;25:10-15.
43. Power TG, O'Connor TM, Orlet Fisher J, et al. Obesity Risk in Children: The Role of Acculturation in the Feeding Practices and Styles of Low-Income Hispanic Families. Child Obes 2015;11:715-21.

44. Nieri T, Lee C, Kulis S, et al. Acculturation among Mexicanheritage preadolescents: A latent class analysis. Soc Sci Res 2011;40:1236-48.

45. Zhang W, Lei K. The Urban New Immigrants' Social Inclusion Internal structure, present situation and influential factors. Sociological Study 2008:117-41.

46. Barona A, Miller J. Short acculturation scale for Hispanic youth (SASH-Y). Hispanic Journal of Behavioral Sciences 1994;16:155-62.

47. Berry J. Acculturation as varieties of adaptation. In: Acculturation: theory, models and some new findings. Westview, 1980:9-25.

48. Mo X. Reflections on the Age Boundary of "Young People". Journal of Guangxi Youth Leaders College 2009;19:38-40.

49. La Greca AM, Dandes SK, Wick P, et al. Development of the Social Anxiety Scale for Children: Reliability and Concurrent Validity. J Clin Child Psychol 1988;17:84-91.

50. Li F, Su L, Jin Y, et al. Norm of the screen for child social anxiety related emotional disorders in Chinese urban children. Chinese Journal of Child Health Care 2006;14:335-7.

51. Shu XO, Zheng W, Potischman N, et al. A population-based case-control study of dietary factors and endometrial cancer in Shanghai, People's Republic of China. Am J Epidemiol 1993:137:155-65.

52. Nichols M, Stein AD, Wold JL. Health status of children of migrant farm workers: farm worker family health program, Moultrie, Georgia. Am J Public Health 2014;104:365-70.

53. Lv N, Cason KL. Dietary pattern change and acculturation of Chinese Americans in Pennsylvania. J Am Diet Assoc 2004;104:771-8.

54. Ma J, Cai CH, Wang $\mathrm{HJ}$, et al. The trend analysis of overweight and obesity in Chinese students during $1985-2010$. Chin J Prev Med 2012;46:776-80.

55. Lu S, Pan D, Liu J, et al. Comparisons of obesity prevalence in children aged 7 to 12 years old in Liaoning ang Guangdong Province. Chin J Sch Health 2017;38:173-6.

56. Boyce T. The media and obesity. Obesity Reviews 2007;8(s1):201-5.

57. Renzaho AMN, Swinburn B, Burns C. Maintenance of traditional cultural orientation is associated with lower rates of obesity and sedentary behaviours among African migrant children to Australia. Int $J$ Obes 2008:32:594-600.

58. Voelker DK, Reel JJ, Greenleaf C. Weight status and body image perceptions in adolescents: current perspectives. Adolesc Health Med Ther 2015;6:149-58.

59. Delavari M, Sønderlund AL, Swinburn B, et al. Acculturation and obesity among migrant populations in high income countries - a systematic review. BMC Public Health 2013;13:458

60. Antin TMJ. Contradictions in food choice and body image: implications for obesity prevention. Dissertations \& Theses Gradworks 2012

61. Shan $X-Y, X i B$, Cheng $H$, et al. Prevalence and behavioral risk factors of overweight and obesity among children aged 2-18 in Beijing, China. Int J Pediatr Obes 2010;5:383-9.

62. Sola AO, Steven AO, Kayode JA, et al. Underweight, overweight and obesity in adults Nigerians living in rural and urban communities of Benue State. Ann Afr Med 2011;10:139-43.

63. Sussner KM, Lindsay AC, Greaney ML, et al. The influence of immigrant status and acculturation on the development of overweight in latino families: a qualitative study. J Immigr Minor Health 2008;10:497-505.

64. Elder JP, Arredondo EM, Campbell N, et al. Individual, family, and community environmental correlates of obesity in latino elementary school children. J Sch Health 2010;80:20-30.

65. Wang Z, Liu M. Influential factors and current situation of extracurriculum p.e. of students of guangzhou middle school. J Harbin Inst of Phy Educ 2008;26:64-6. 